\title{
Terminal-Zone Corrections for a Dipole Driven by a Two-Wire Line ${ }^{1}$
}

\author{
Keigo Iizuka and Ronold W. P. King \\ Contribution From Gordon McKay Laboratory, Harvard University, Cambridge, Mass.
}

(Received May 3, 1962; revised June 7, 1962)

\begin{abstract}
The terminal-zone effects on the dipole antenna driven by a two-wire transmission line have been re-examined. A series inductive correction $L_{g}$ together with other terminal-zone corrections is found necessary to reduce the measured apparent admittance of the antenna terminating the two-wire line with the ideal admittance of the dipole antenna when driven by a delta-function generator. The series inductive correction takes account of the absence of the antenna wire in the gap between the two wires of the transmission line.

With this additional correction, the measured apparent admittance of a dipole antenna terminating a closely spaced two-wire line may be brought into excellent agreement with ideal theoretical values and also reconciled with the quite different values measured with a coaxial line for an antenna consisting of the vertical inner conductor of the line extended over a horizontal ground plane. The results further show the utility of a delta-function generator in the definition of an ideal theoretical impedance of a dipole antenna.
\end{abstract}

\section{Introduction}

Theoretical analyses of the center-driven dipole antenna and of the base-driven half dipole erected vertically on an infinite perfectly conducting ground screen make use of idealized generators that eliminate the transmission line that is usually present in practice. Recent work by Duncan and Hinchey [1960] and $\mathrm{Wu}[1961]$ has postulated a discontinuity in the form of a delta-function in the tangential electric field at the driving point in the manner implicit in earlier work by Hallén [1938], King and Middleton [1946], and others. Chen and Keller [1962] have preferred a rotationally symmetrical electric field maintained in an unspecified way across a gap of finite width in the manner of Synge [1948], Infeld [1947], and King and Winternitz [1947]. Theoretical admittance of an antenna driven by either of these physically unavailable generators differs significantly from the apparent admittance of an antenna when connected as the terminating load for a physically available transmission line such as a coaxial line or a two-wire line. A rigorous solution of the two simultaneous integral equations for the currents in a dipole anntena and in a feeding twowire line is still unavailable.

For line spacings that are not too large a fraction of a wavelength, a reasonably satisfactory approximate method has been devised IKing, 1956a; King, 1955b; King, 1949 ; King, 1955c] which introduces a terminalzone network to be used in conjunction with the ideal admittance of the antenna and an ideal transmission line. By the ideal admittance $Y_{0}$ of the antenna is meant the admittance determined with a delta-

1 This research was supported through Joint Services Contract No. Nonr 1866(32) between Harvard University, the Office of Naval Research, the Signal $1866(32)$ between Harvard University, the Office
Corps of the U.S. Army, and the U.S. Air Force function generator after the infinite susceptance due to the knife edges implicit in such a generator has been subtracted out - an analytically sophisticated process discussed in detail in the literature, [Wu and King, 1959; Duncan, 1962]. An ideal transmission line is one with uniform line parameters even near the load. The terminal-zone network takes account of transmission-line end effects, of coupling between the antenna and the line, and of any other characteristics of a small region near the junction of the transmission line and the antenna.

An extensive systematic experimental study of the effect of the cross-sectional size of a coaxial line on the apparent impedance of the vertical monopole that it drives over a ground screen was made by D. D. King [1946] and Hartig [1950]. (The earlier work of Brown and Woodward [1945] showed the effect of lumped positive capacitances in parallel with the antenna, but did not refer to the negative capacitive junction effect determined by the size of the coaxial line.) Their measurements are in quite good agreement with theoretical results obtained with a delta-function generator when a suitable corrective network is used. Moreover, they indicate that the measured apparent admittance $Y_{s a}$ of a half dipole that consists of the extended inner conductor (radius $a$ ) of a coaxial line (inner radius of outer conductor $b$ ) approaches the theoretical admittance $Y_{\mathrm{o}}$ derived with a delta-function generator as the ratio $b / a$ approaches one.

Although the terminal-zone problem of a dipole antenna when center-driven by a two-wire line (that consists of conductors of radius $a$ separated by a distance $b$ between centers) has been studied extensively [King, 1956a; King, 1955b; King, 1949; King and King, D. D., 1945; Essen and Oliver, 
1945], attention has been focused primarily on transmission-line end effect and coupling between antenna and line, which are the major contributors to the difference between $Z_{s a}$ and $Z_{0}$ in the case of the monopole driven by a coaxial line over a ground screen. The significance of the gap at the center of the dipole when driven by a two-wire line-which has no counterpart in the monopole that consists of the extended and unbroken inner conductor of a coaxial line has not been investigated experimentally in any definitive manner. A theoretical study of this gap is available [King and Winternitz, 1947], but a detailed comparison of its predictions of its measured values has not been made.

In order to compare the measured driving-point admittance of an antenna terminating a two-wire line with the ideal admittance of a dipole antenna when driven by a delta-function generator, account must be taken of the following three effects:

(a) Deviation of the inductance and capacitance per unit length and hence, of the characteristic impedance of a two-wire line of finite length near its end from the corresponding quantities characteristic of the infinitely long line.

(b) Electromagnetic coupling between the transmission line and the antenna near their junction.

(c) The absence of the conductor in the gap in the antenna between the two wires of the transmission line.

The effects under (a) and (b) have been treated in the literature [King, 1956a; King, 1955b]. The effect (c) has been studied theoretically [King and Winternitz, 1947], but has not been included in a corrective network. This report is devoted primarily to a study of this important correction which may be surprisingly large, for the dimensions of the apparatus used in the measurements to be described, the correction for (c) was three times as large as that for (b).

In the investigation three different methods were used and their final results compared. They are:

(1) Repeated measurements were made with progressively reduced spacing $b$ of the two-wire transmission line which fed the dipole antenna. The measured values of the apparent admittance terminating the line were extrapolated to determine the ideal limiting value for zero spacing, i.e., for $b=2 a$.

(2) The correction under (c) was calculated by simply taking the difference in the inductive reactance between the antenna with half length $h$ and that with half-length $h-b / 2$. The distance from one end of the antenna to the other is $2 h$. The measured driving point admittance corrected for all the effects (a), (b), and (c) was compared with the King-Middleton second-order theory.

(3) The measured apparent admittance of the dipole antenna with a gap was corrected according to Winternitz's theory [King and Winternitz, 1947] and then compared with the King-Middleton second-order theory.

It was verified that the corrections applied in (2) and (3) are essentially the same. The zeroth-order correction in (3) could be reduced to the results of (2).

\section{Experimental Arrangement and Measured Admittances}

A detailed description of the apparatus used for measuring the admittance of a dipole antenna driven by a two-wire transmission line has been given elsewhere [Iizuka, King, and Prasad, 1962]. The two-wire line and the dipole antenna were made of brass tubing with radius $a=0.318 \mathrm{~cm}$. The spacing $b$ of the line was maintained at one of the values, $b=0.79 \mathrm{~cm}, b=1.27 \mathrm{~cm}, b=3.11 \mathrm{~cm}$ by means of polyfoam spacers arranged along the transmission line. A continuous wave with a wavelength of 36 $\mathrm{cm}(833.33 \mathrm{Mc} / \mathrm{s})$ was used. The apparent terminal admittance of the dipole at the end of the line was measured for electrical half lengths in the range from $\beta h=0.7$ through 6.8 (where $\beta=2 \pi / \lambda$ ). These measurements were repeated for each of the three different spacings. The corresponding parameters are listed in table 1.

TABLE 1. Parameters of the two-wire transmission line

\begin{tabular}{|c|c|c|c|c|}
\hline Case & Spacing & $b / a$ & $\boldsymbol{\beta} b$ & $Y^{*}{ }_{0}$ \\
\hline $\begin{array}{l}\text { I } \\
\text { II } \\
\text { III }\end{array}$ & $\begin{array}{l}c m \\
0.79 \\
1.27 \\
3.11\end{array}$ & $\begin{array}{l}2.5 \\
4.0 \\
9.0\end{array}$ & $\begin{array}{l}\text { Rad } \\
0.138 \\
.222 \\
.543\end{array}$ & $\begin{array}{r}\text { Millimho } \\
12.02 \\
6.33 \\
3.79\end{array}$ \\
\hline
\end{tabular}

${ }^{*} Y_{\mathrm{o}}$ is the characteristic admittance of the two-wire transmission line.

In figure 1 , the measured apparent terminal admittances as measured on a line with $b / a=4.0$ are compared without modification or correction with the King-Middleton 2d-order theory [King, 1955b] and Wu's theory [1961] as a function of the electrical half length $\beta h$. These admittances are converted into the equivalent impedances shown in figure 2.

It is observed in figure 1 that the amplitudes of the oscillation of the experimental curves of both the conductance $G$ and the susceptance $B$ are not only smaller than those of the theoretical curves, but also that both of the experimental curves are shifted slightly towards larger values of $\beta h$. The entire measured susceptance curve is much lower than that predicted by theory. Similar observations can be made with regard to the impedance curves in figure 2 except that the amplitudes of the oscillations of the experimental curves of both the resistance $R$ and the reactance $X$ are larger than given by theory. From these curves, it should be clear that a mere plotting together of theoretical and experimental results may be very misleading. The measured apparent admittance includes all end effects which can be approximated by a shunt lumped susceptance $\Delta B$, and a lumped series reactance $\Delta X$. In effect, $\Delta B$ moves the entire susceptance curve vertically while $\Delta X$ contracts the amplitudes of oscillation of the measured apparent admittance $Y_{s a}=G_{s a}+j B_{s a}$. The reason is as follows: the apparent admittance is given in terms of the ideal antenna impedance $R$ and $X$ :

$$
\begin{gathered}
G_{s a}=\frac{R}{R^{2}+(X-\Delta X)^{2}} \\
B_{s a}=-\frac{X}{R^{2}+(X-\Delta X)^{2}}+\Delta B .
\end{gathered}
$$




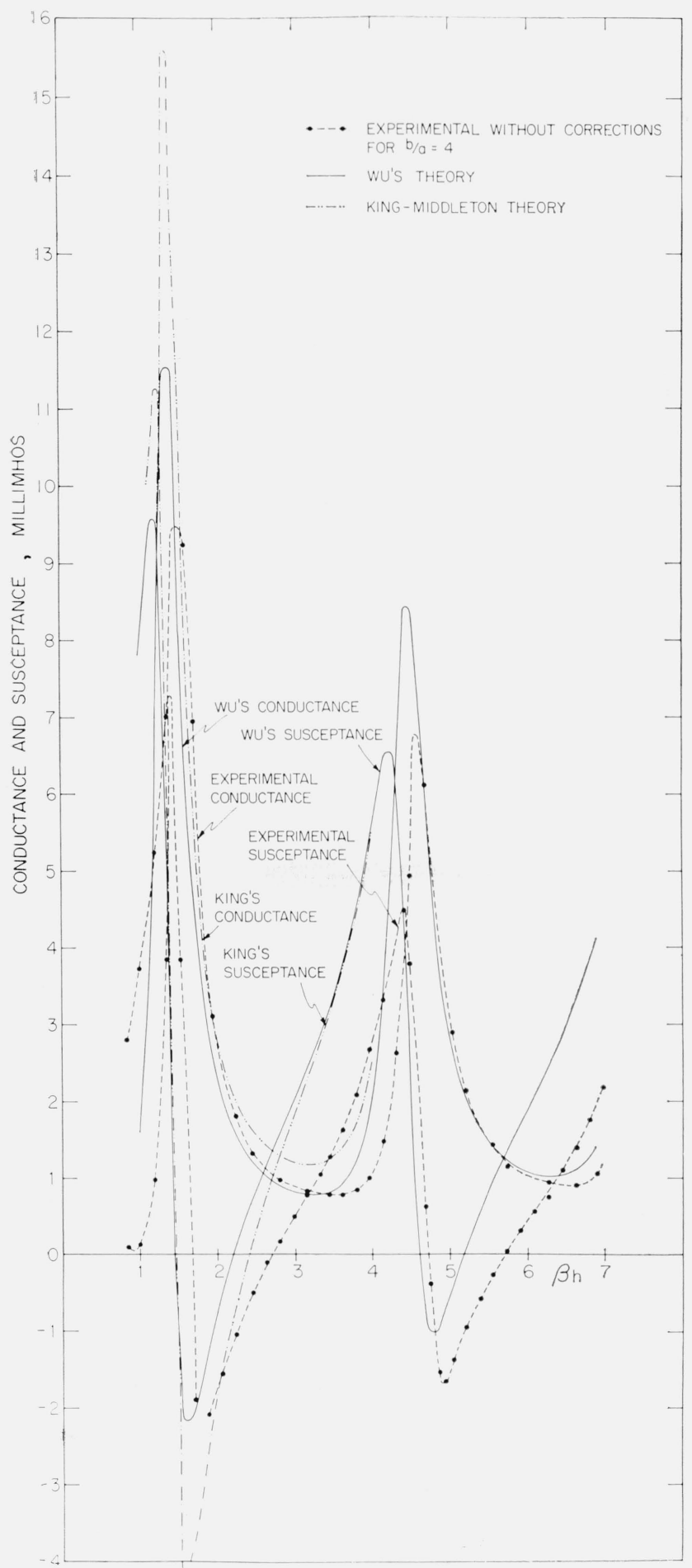

Figure 1. Measured driving-point admittance of the dipole antenna driven by the two-wire line of $\mathrm{b} / \mathrm{a}=4$ without corrections.

First, it is obvious that $\Delta B$ slides the entire susceptance curve by the amount $\Delta B$. Second, it is seen from (1) and (2) that the value of $\beta h$ which gives the maximum $G_{s a}$ is shifted from the vicinity of $\beta h_{r}$ to that of $\beta h_{s}$ where $X\left(\beta h_{r}\right)=0$ and $X\left(\beta h_{s}\right)=\Delta X$, and

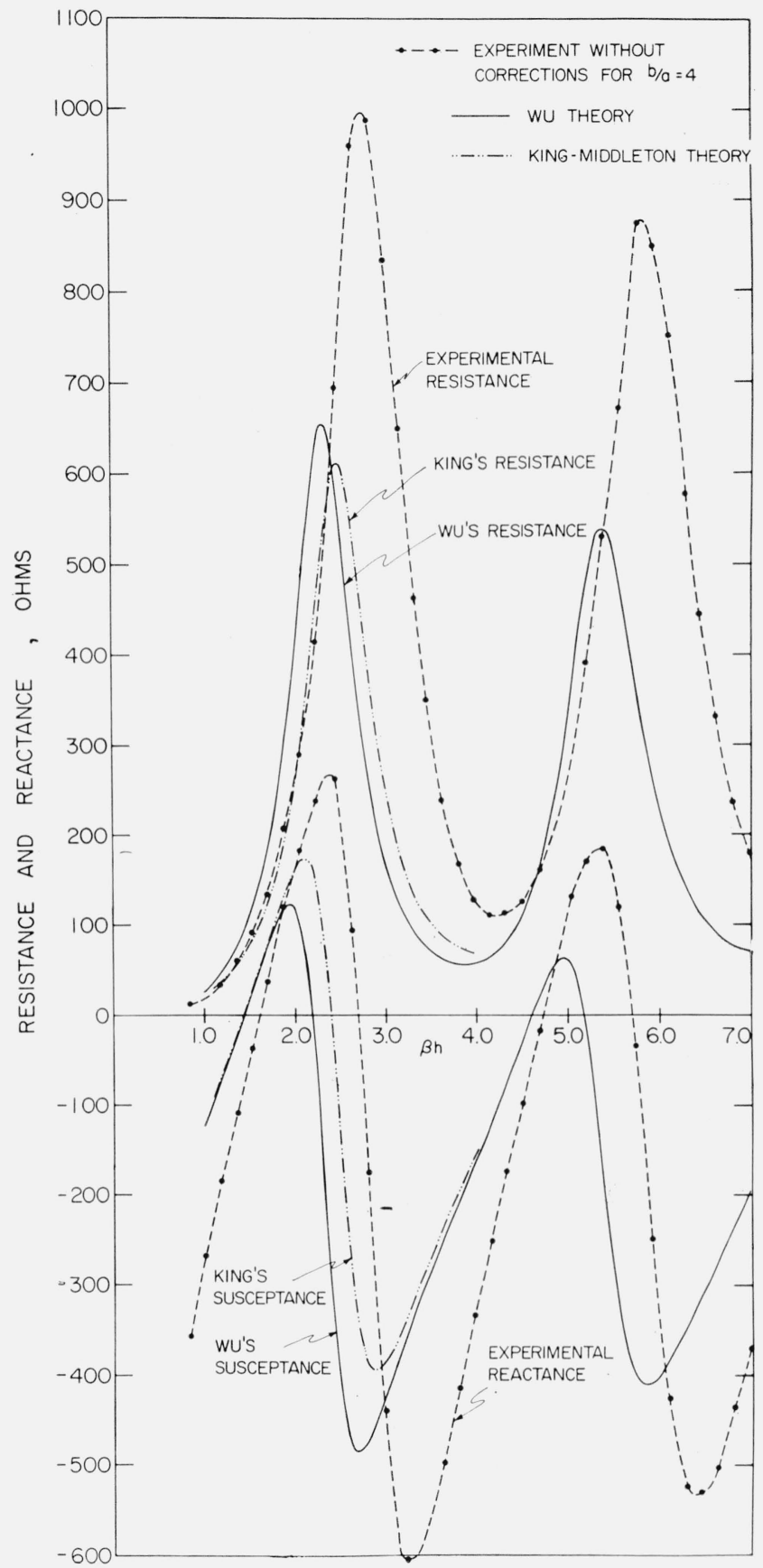

Figure 2. Measured driving-point impedance of the dipole antenna driven by the two-wire line of $\mathrm{b} / \mathrm{a}=4$ without corrections.

the value of the maximum becomes

$$
\left(G_{s a}\right)_{\max }=\frac{1}{R\left(\beta h_{s}\right)}
$$

and $R\left(\beta h_{s}\right)>R\left(\beta h_{r}\right)$ or $\left(G_{s a}\right)_{\max }<G_{\max }$. Hence, the amplitude of the oscillations of the measured apparent conductance is smaller than that of the ideal conductance. The susceptance behaves in a similar manner. 


\section{Terminal-Zone Corrections}

The modifications made in many instances on the measured driving-point admittance of the dipole antenna driven by a two-wire transmission line have been due to (A) the change of the characteristic impedance of the two-wire transmission line toward the end of the line, (B) the electrical coupling between the antenna and transmission line. The inductive part of the effect (A) can be approximated by a lumped series inductor $L_{T}$ and capacitive part of (A) together with the effect (B) by the lumped shunt capacitor $C_{T}$ (note that there is no inductive coupling between the antenna and the transmission line since the antenna and the line are mutually perpendicular). Approximate formulas for $L_{T}$ and $C_{T}$ are found in King [1955b]. The values of $C_{T}, \omega C_{T}, L_{T}$, and $\omega L_{T}$ appropriate for the present case are in table 2.

TABLE 2. Lumped elements for terminal-zone corrections

\begin{tabular}{|c|c|c|c|}
\hline & $b / a=2.5$ & $b / a=4$ & $b / a=9$ \\
\hline $\begin{array}{l}L_{T} \\
\omega_{T} L_{T-1} \\
C_{T} \\
\omega C_{T}\end{array}$ & $\begin{array}{l}-0.95 \times 10^{-9} \\
-5.0 \\
-0.56 \times 10^{-12} \\
-2.9 \times 10^{-3}\end{array}$ & $\begin{array}{l}-1.9 \times 10^{-9} \\
-10.0 \\
-0.28 \times 10^{-12} \\
-1.5 \times 10^{-3}\end{array}$ & $\begin{array}{l}-5.1 \times 10^{-9} \\
-26.6 \\
-0.26 \times 10^{-12} \\
-1.4 \times 10^{-3}\end{array}$ \\
\hline
\end{tabular}

The experimental results were modified in the conventional manner using the results in table 2 . The measured admittance with corrections, $\omega L_{T}$ and $\omega C_{T}$ are shown in figure 3 and the equivalent impedances in figure 4 . It is seen in figure 3 that the extreme values of both $G$ and $B$ are still smaller, especially in the range $\beta_{h}<2 \pi$ than the corresponding King-Middleton theoretical results. The shift of the entire curve toward larger values of $\beta h$ is still evident. These partially corrected experimental results might be interpreted to provide an excellent verification of $\mathrm{Wu}$ 's theory at the shorter lengths rather than of the King-Middleton 2d-order theory, even though the asymptotic nature of Wu's analysis suggests decreasing accuracy as the length of the antenna is reduced, and the King-Middleton results have been verified by measurements with a coaxial line [King, D. D., 1946, Hartig, 1950]. Evidently, an additional correction is required to take account of the fact that the antenna is not a continuous conductor of length $2 h$ with a discontinuity in scalar potential at the center but has a spacing $b$.

\section{Measurements With Progressive Spacing of the Two-Wire Line}

A simple and convincing experiment to show the necessity of an additional correction to take account of the absence of the antenna wire in the gap between the two wires of the transmission line consists in the repeated measurement of the admittance of the antenna as the spacing of the conductors of the twowire line is decreased. The measured admittance with three spacings when corrected for the shunt capacitances $C_{T}$ in table 2 is shown in figure 5 .

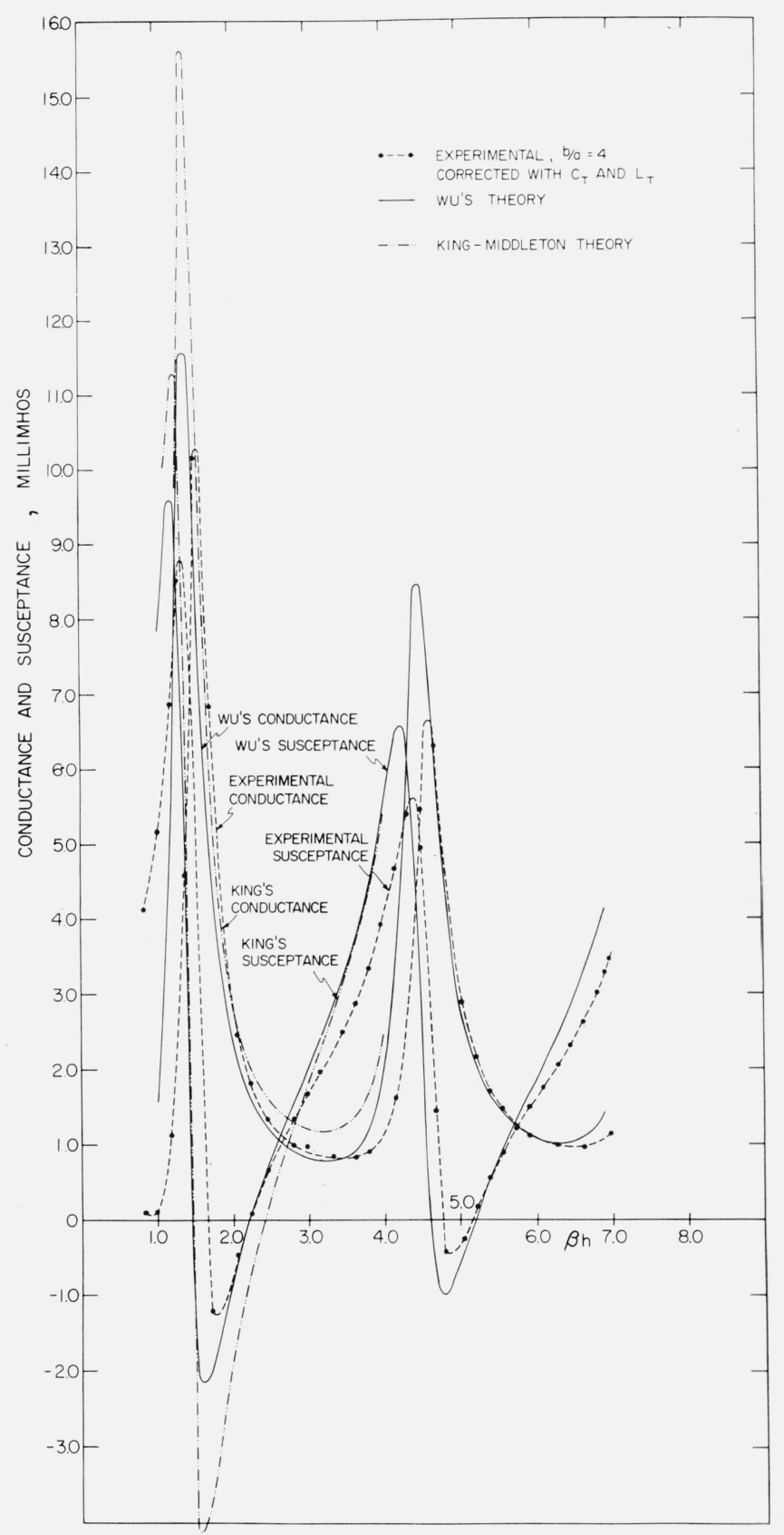

FIGURE 3. Measured driving-point admittance of the antenna corrected in the conventional way with $\mathrm{b} / \mathrm{a}=4$.

Since the present purpose is to obtain the extrapolated values of admittance, there is no necessity to make a correction for $L_{T}$ which becomes smaller with a decrease in spacing: $L_{T}=-\frac{b-a}{2 \pi \nu}$ and in the limit at $b=2 a$ is only $L_{T}=-\frac{a}{2 \pi \nu}=-3$ ohms. The line spacings were $b / a=2.5, b / a=4$, and $b / a=9$. (The widest spacing, $b / a=9$, is rather large for the two-wire line to be considered as a conventional transmission line, since the value of $\beta b$ for this case 


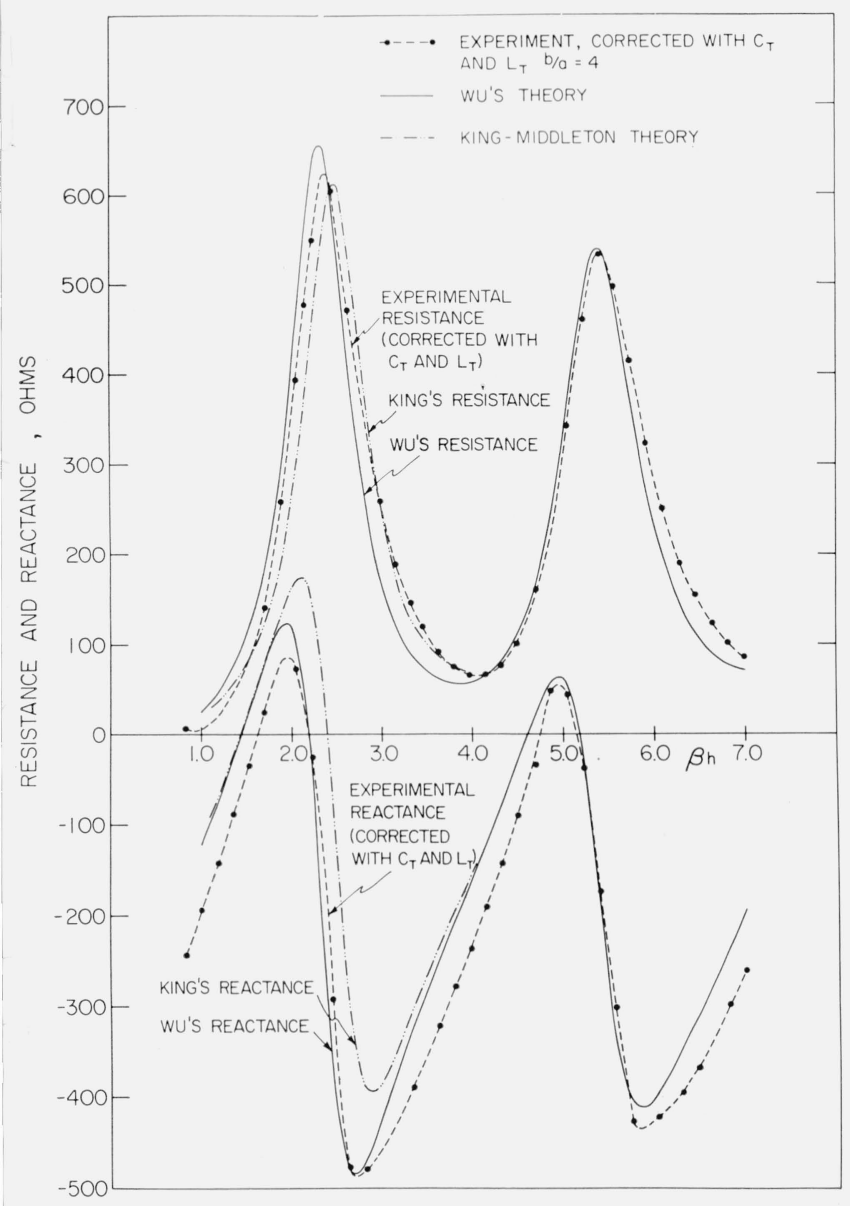

Figure 4. Measured driving-point impedance of the antenna corrected in the conventional way with $\mathrm{b} / \mathrm{a}=4$.

is $\beta b=0.54$ which does not satisfy the condition

$$
\beta b \ll 1
$$

imposed in the reduction of the Maxwell's equations to the telegraphist's equations [King, 1955a].) It is observed that as the spacing of the wires was reduced, the oscillation in the amplitudes of both the conductance and the susceptance curves increased and approached the curve of the KingMiddleton 2d-order theory. In order to make the extrapolation, the conductance was replotted in rectangular coordinates as a function of the spacing ratio $b / a$ in figure 6 (a) and the susceptance in figure 6(b). The conductance and susceptance curves of the extrapolated values are compared with KingMiddleton $2 d$-order theory in figures 7 (a) and $7(b)$. Note that when account is taken of the proximity effect, [King, 1945], the extrapolation must be taken to $b / a=2$, which is the limit where the transmission lines make contact with each other (not to the value $b / a=0)$.

Both conductance and susceptance curves which were extrapolated to the spacing $b / a=2$ show good

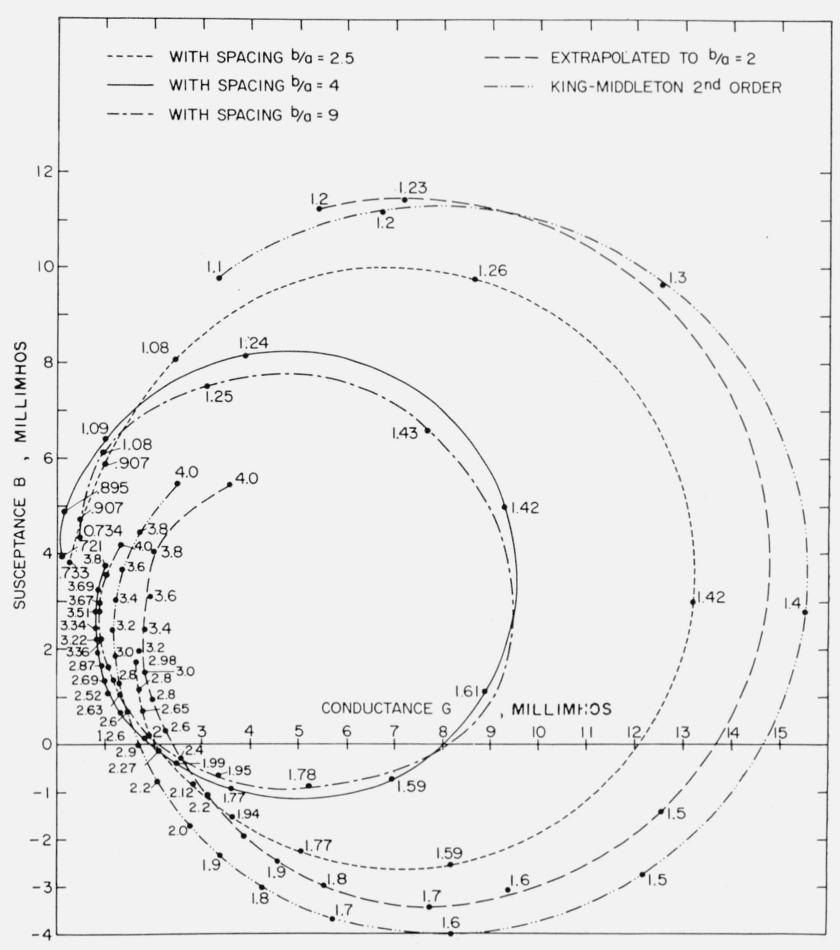

FIGURE 5. Driving-point admittance of the antenna with various spacings of the two-wire transmission line.
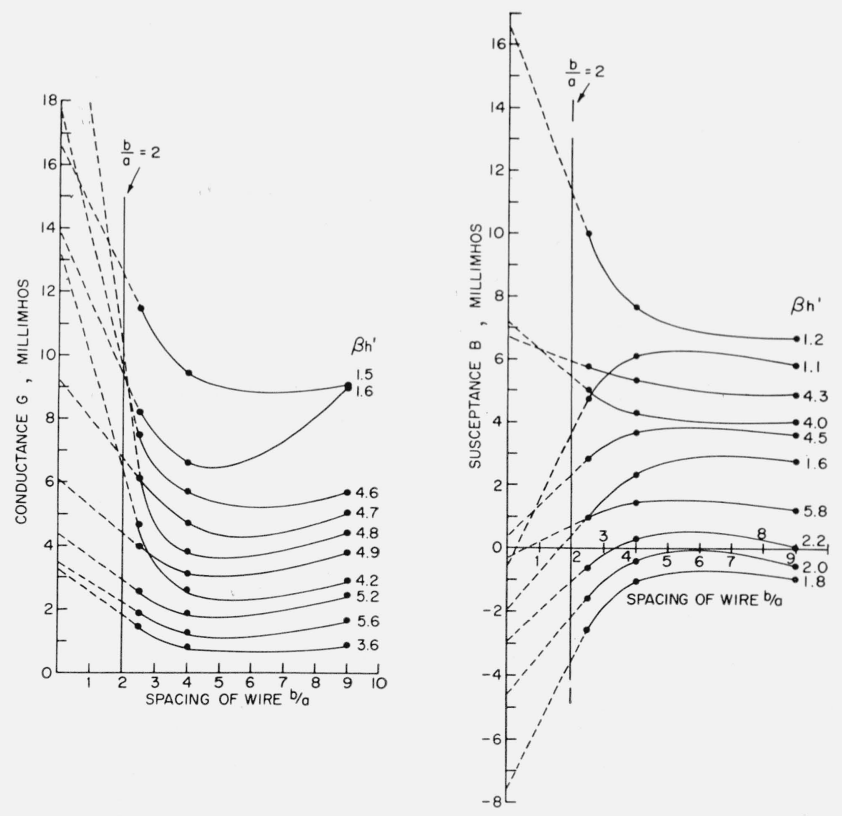

(a)

(b)

Figure 6. The extrapolation of the measured driving-point admittance of the antenna.

agreement with the theory except that the extrapolated conductance and susceptance are both slightly smaller near resonance and slightly larger near antiresonance. 

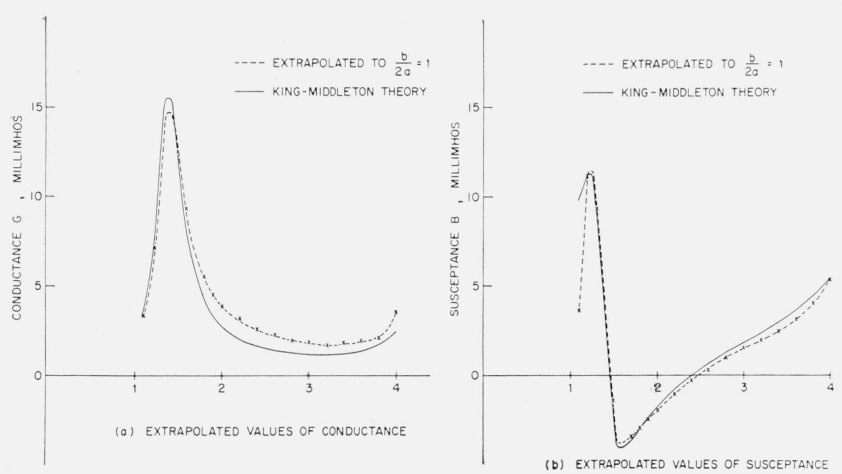

FigURE 7. Extrapolated values of the admittance of the antenna.

\section{Zero-Order Correction for the Gap at the Driving Point}

The lumped series inductance $L_{g}$ required to correct the gap at the driving point may be calculated from a zero-order theory. The emf $V$ of a deltafunction generator that maintains the current $I$ at the center of an antenna of half length $h$ is to the zero-order approximation [King, 1956b].

$$
V=-j \frac{\xi_{0} \Psi}{2 \pi} I \cot \beta_{0} h .
$$

If the same antenna is driven with a small separation $b / 2$ at the center and the current is to remain the same as that of an antenna driven by a delta-function generator, the driving voltage must be different. To the zero-order, it should be

$$
V_{b / 2}=-j \frac{\zeta_{0} \Psi}{2 \pi} I \cot \beta_{0}\left(h-\frac{b}{2}\right)
$$

The difference $\Delta V$ between (5) and (4) is

$\Delta V=V_{b / 2}-V=-j \frac{\zeta_{0} \Psi}{2 \pi} I\left\{\cot \beta_{0}\left(h-\frac{b}{2}\right)-\cot \beta_{0} h\right\}$.

In other words, if a lumped impedance, $\frac{\Delta V}{I}=j \omega L_{g}$, is inserted in eries with the antenna when its driving terminals are separated by a distance $b$, the current distribution along the antenna is the same as when the antenna is driven by a delta-function generator driven by the same voltage.

The reactance $\dot{X}_{g}=\omega L_{g}$ is given by

$$
X_{g}=\omega L_{g}=-\frac{\zeta_{0} \Psi}{2 \pi}\left\{\cot \beta_{0}\left(h-\frac{b}{2}\right)-\cot \beta_{0} h\right\} \text {. }
$$

The value of $\omega L_{g}$ near the resonant length of the antenna is calculated, for instance, for case II in table 1 to be

$$
X_{g}=\omega L_{g}=-30.6 \text { ohms. }
$$

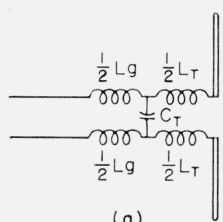

(a)

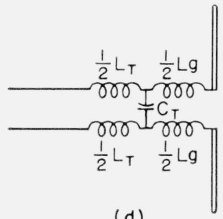

(d)

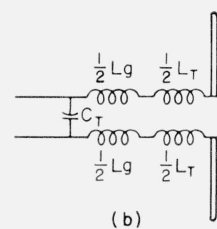

(b)

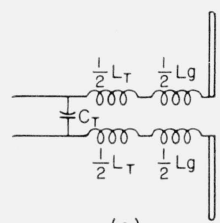

(e)

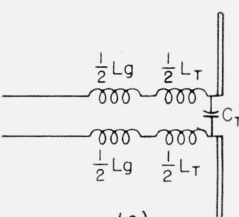

(c)

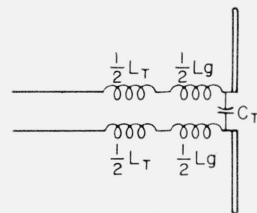

(f)

Figure 8. Possible equivalent networks for the antenna driven by a two-wire line.
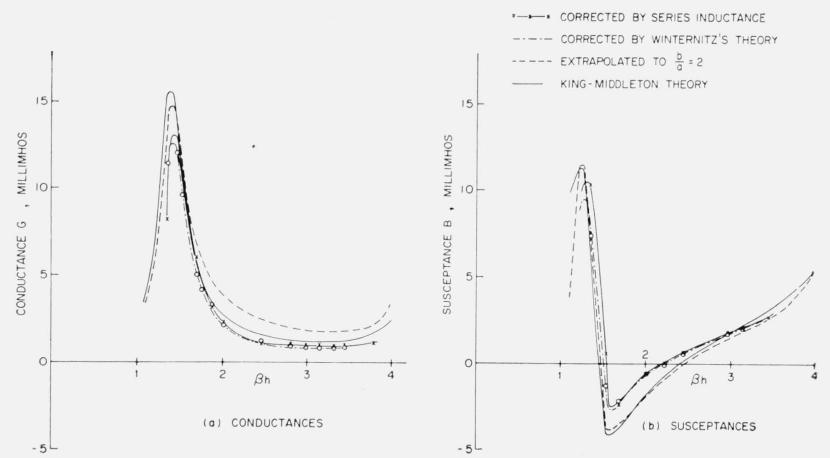

Figure 9. Measured admittance curves corrected in three different ways.

This is the lumped series reactance required to correct for the gap; it must be combined with the corrections $j \omega C_{T}$ and $j \omega L_{T}$ mentioned above. Of the six possible combinations for connecting $C_{T}, L_{T}$, and $L_{g}$ shown in figure 8 , the equivalent circuit $a$ in figure 8 seems preferable from the physical point of view. However, convenience in computation makes the equivalent circuit $b$ or $e$ a better choice. Note that the use of lumped elements in a corrective network is a useful approximation only if the differences in the use of the equivalent circuits in figure 8 are negligible.

The apparent driving-point admittance with corrections for $C_{T}, L_{T}$, and $L_{g}$ is shown in figure 9 In comparison with the admittance curves corrected only with $C_{T}$ and $L_{T}$ as shown in figure 4 , the curves with all three corrections shown in figure 9 are in much better agreement with the King-Middleton theory.

\section{Evaluation of $L_{g}$ by Winternitz's Theory}

A Maclaurin expansion of the expression for the driving-point admittance $Y_{\delta}$ of the antenna with the base separation $b=2 \delta$ in the vicinity of $\beta \delta=0$ allows the impedance of the antenna to be expressed in terms of the admittance $Y_{0}$ of the antenna driven 
by a delta-function generator [King and Winternitz 1947], [King, 1956c]. Thus, when $\beta \delta$ is small,

$$
Y_{\delta}=Y_{0}+\beta \delta\left[\frac{\partial Y_{\delta}}{\partial\left(\beta_{0} \delta\right)}\right]_{j=0}=Y_{0}(1-\epsilon)
$$

where

$$
\epsilon=\epsilon^{\prime \prime}-j \epsilon^{\prime}=-\frac{\beta_{0} \delta}{Y_{0}}\left[\frac{\partial Y \delta}{\partial(\beta \delta)}\right]_{j=0}
$$

or

$$
\begin{aligned}
& G_{\delta}=G_{0}\left(1-\epsilon^{\prime \prime}\right)-B_{0} \epsilon^{\prime} \\
& B_{\delta}=B_{0}\left(1-\epsilon^{\prime \prime}\right)+G_{0} \epsilon^{\prime}
\end{aligned}
$$

values of $\epsilon^{\prime \prime}$ and $\epsilon^{\prime}$ for different thicknesses of the antenna were computed by [King and Winternitz 1947]. The curves of $\frac{h \epsilon^{\prime \prime}}{\delta}$ versus $\beta h$ and $\frac{h \epsilon^{\prime}}{\delta}$ versus $\beta h$, and the curves of $R_{0}-R_{g}$ versus $\beta h$ and $X_{0}-{ }_{g} X$ versus $\beta h$ are also available [King and Winternitz, 1947; King, 1956c].

The measured apparent driving-point admittance for a line with $b / a=4$ was corrected with values obtained from the curves of $R_{0}-R_{g}$ and $X_{0}-X_{g}$ versus $\beta h$ for $\Omega=21 \mathrm{n}(2 h / a)=10$. (The value of $\mathrm{n}$ for the antenna used in the experiment ranged from $\Omega=7.3$ to 9.5 .) The results are shown in figure 9 together with the curves obtained by the other methods of correction. Actually, and as is readily verified, the correction made in section 5 is the same as that given by (11) if the zero-order admittance is used in the correction term.

\section{Comparison of the Corrections for the Gap Made in Different Ways}

The admittance curves obtained in three different ways are all shown in figure 9 together with the results of the King-Middleton theory. It is seen that the curves of both the conductance and the susceptance which were obtained empirically by extrapolation to zero line spacing show the best agreement with the theory. The extrapolated conductance, however, is larger than given by the theory in the range between $\beta h=1.8$ and $\beta h=3.0$. The extrapolated curves of the conductance and susceptance show an excellent agreement with the theory near resonance; the curves obtained by either the series inductance $L_{g}$ correction or the Winternitz $2 \mathrm{~d}$-order correction yield smaller values than the theory in this region. Both the conductance and susceptance curves obtained by the relatively simple series inductance $L_{g}$ correction are not very different from those of the more complicated Winternitz $2 d$-order theory except in the small range near resonance.

\section{Conclusions}

It has been shown that the difference between the measured apparent admittance of (1) a dipole antenna driven by a two-wire line, and (2) a monopole antenna driven from a coaxial line over a ground plane, is due in large part to the absence of the conductance between the terminals of the dipole. It has also been shown that a series inductance $L_{g}$ may be evaluated to correct this difference approximately. Alternatively and somewhat more accurately, a correction may be made empirically if measured values are extrapolated to "zero" line spacing. Apparent admittances corrected either theoretically with a suitable terminal-zone network of lumped elements $L_{g}, C_{T}$, and $L_{T}$ or empirically, by extrapolation are shown to be in good agreement with theoretical values obtained with a hypothetical delta-function generator without knife-edge capacitance.

The authors are indebted to fruitful discussions with Professor T. T. Wu.

\section{References}

Brown, G. H., and O. M. Woodward, Experimentally determined impedance characteristics of cylindrical antennas. Proc. IRE 33, 257-262 (1945).

Chen, Y. M., and J. B. Keller, Current on and input impedance of a cylindrical antenna, J. Research NBS 66D (Radio Prop) No. 1, 15-21 (Jan.-Feb. 1962).

Duncan, R. H., Theory of the infinite cylindrical antenna including the feed-point singularity in the antenna current, J. Research NBS 66D (Radio Prop.) No. 2 181-188 (Mar.-Apr. 1962).

Duncan, R. H., and F. A. Hinchey, Cylindrical antenna theory, J. Research NBS 64D (Radio Prop.) 569-584 (1960).

Essen, L., and M. H. Oliver, Aerial impedance measurements, Wireless Engr. 22, 587 (1945).

Hallén, E., Theoretical investigations into transmitting and receiving antennae, Nova Acta Regiae Soc. Sci. Upsaliensis (4) 11, 1 (1938).

Hartig, E. O., Circular apertures and their effects on halfdipole impedances, Doctoral Dissertation, Harvard University, 1950. (Reprinted in reference [King, 1956], pp. $231-237$.

Iizuka. K., R. W. P. King, and S. Prasad, The long, cylindrical antenna: current and admittance, Cruft Laboratory Tech. Rept. 351 (1962).

Infeld, L., The influence of the width of the gap upon the theory of antennas, Quart. Appl. Math. 5, 113 (1947).

King, D. D., The measured impedance of cylindrical dipoles, J. Appl. Phys. 17, 844 (1946).

King, R. W. P., Electromagnetic engineering, pp. 435-441 (McGraw-Hill Book Co., Inc., New York, 1945).

King, R. W. P., and D. D. King, Microwave impedance measurements with application to antennas II, J. Appl. Phys. 16, 445 (1945).

King, R. W. P., and D. Middleton, The Cylindrical antenna: Current and impedance, Quart. Appl. Math. 3, 302-335 (1946).

King, R. W. P., Antennas and open-wire lines I, J. Appl. Phys. 20, 832 (1949).

King, R. W. P., Transmission line theory, p. 23 (McGrawHill Book Co., Inc., New York, 1955a).

King, R. W. P., Transmission line theory, pp. 405-411, 430437 (McGraw-Hill Book Co., Inc., New York, 1955b). 
King, R. W. P., End correction for a coaxial line when driving an antenna over a ground screen, Trans. IRE Ant. Prop. $\mathbf{A P}-\mathbf{3}, 66$ (1955c).

King, R. W. P., Theory of linear antennas, pp. 31-69, 193-237 (Harvard Univ. Press, Cambridge, Mass., 1956a).

King, R. W. P., Theory of linear antennas, p. 86 (Harvard Univ. Press, Cambridge, Mass., 1956b).

King, R. W. P., Theory of linear antennas, pp. 149, 150, 194-197 (Harvard Univ. Press, Cambridge, Mass., 1956c). King, R. W. P., and T. W. Winternitz, The cylindrical antenna with gap, Quart, Appl. Math. 5, 403 (1947).
Synge, J. L., The general problem of antenna radiation and the fundamental integral equation, with application to an antenna of revolution, Quart. Appl. Math. 6, 133 (1948).

Wu, Tai Tsun, Theory of the dipole antenna and the two-wire transmission line, J. Math. Phys. 2, 550-574 (1961).

Wu, Tai Tsun, and R. W. P. King, Driving point and input admittance of linear antennas, J. Appl. Phys. 30, 74-76 (1959).

(Paper 66D6-235) 